\title{
Echocardiographic demonstration of Escherichia coli endocarditis restricted to the pulmonary valve
}

\author{
NEIL H MURRAY, MARK G CHEESMAN, * MICHAEL MILLAR-CRAIG
}

From the Department of Cardiology, Groby Road Hospital, Leicester

SUMMARY A 25 year old man with no history of heart disease presented with sweats and rigors. Echocardiography showed a large vegetation on the pulmonary valve and blood cultures grew Escherichia coli. Because of recurrent pulmonary emboli a large vegetation on the anterior leaflet of the pulmonary valve was excised. He recovered after a full course of antibiotics.

Requests for reprints to Dr Neil H Murray, Department of Cardiology, Regional Cardiothoracic Unit, Groby Road Hospital, Leicester LE3 9QE.

*Present address: Department of Medicine, University Hospital of Wales, Heath Park, Cardiff.

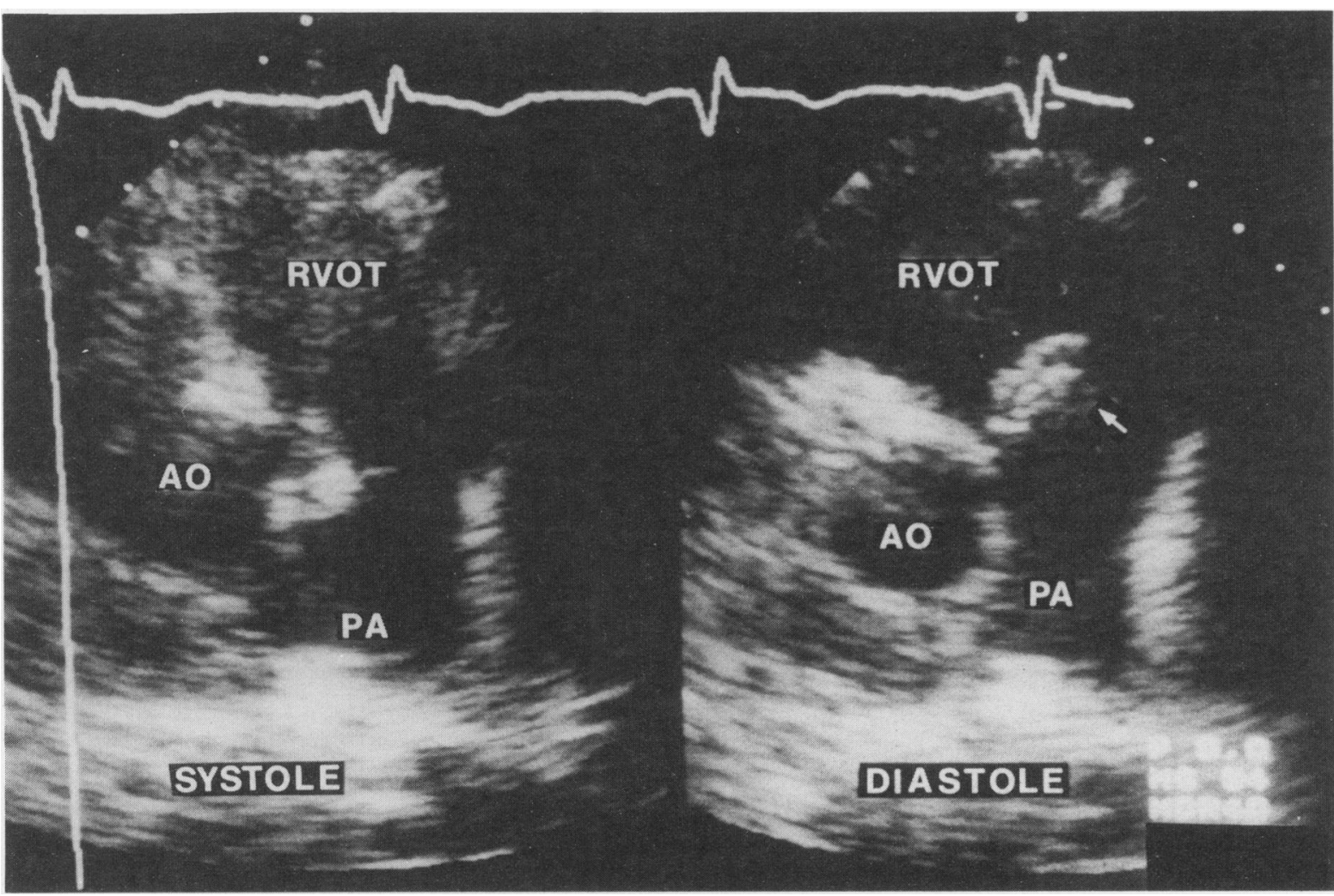

Fig 1 Parasternal short axis view showing a large vegetation on the pulmonary valve (arrow). RVOT, right ventricular outflow tract; $P A$, pulmonary artery; $A O$, aorta.
Isolated endocarditis of the pulmonary valve is uncommon and rarely occurs on native valves if there are no predisposing factors such as congenital heart disease or intravenous drug abuse. The pulmonary valve is also the most difficult of the four cardiac 


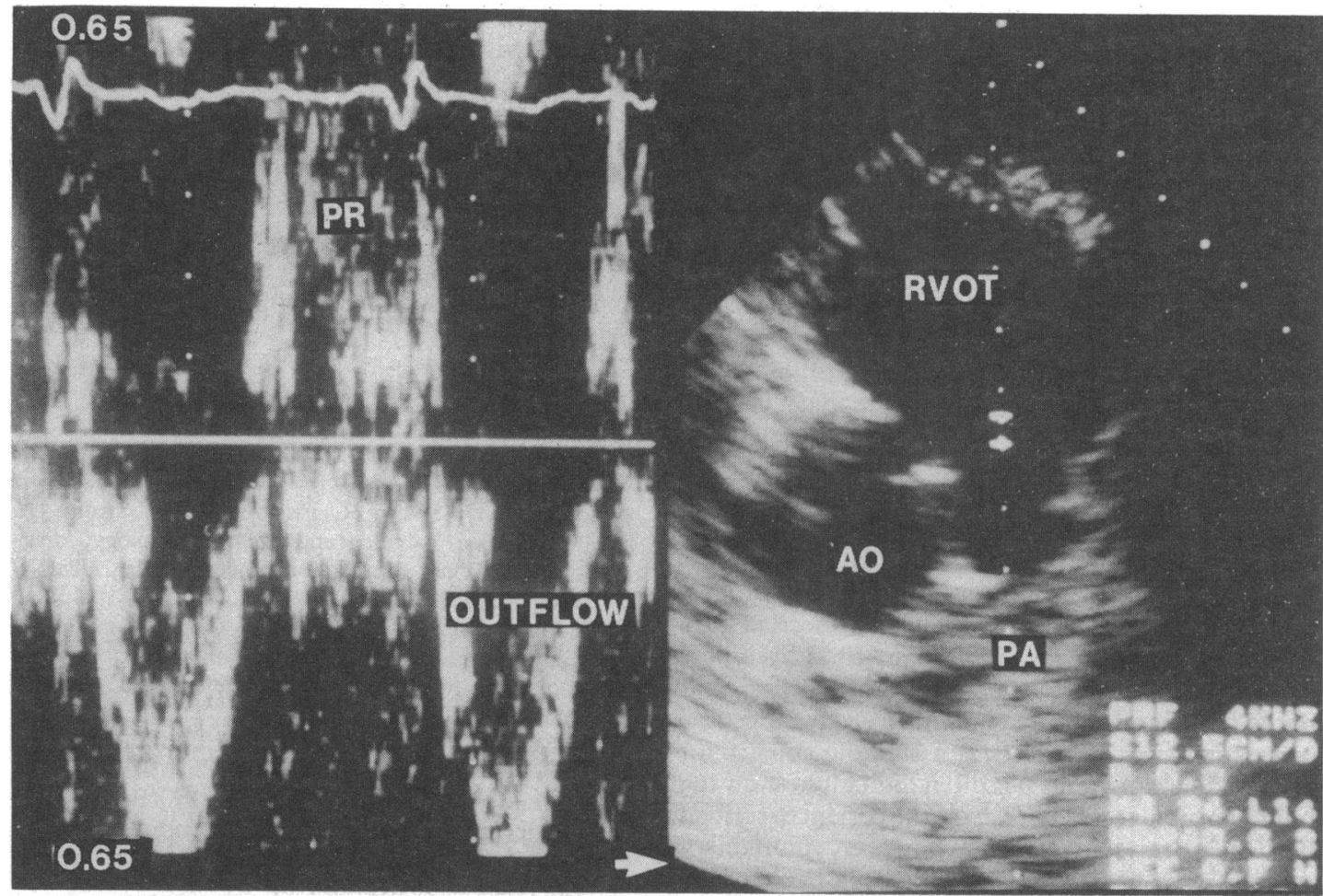

Fig 2 Parasternal short axis view with pulsed wave Doppler ultrasound showing pulmonary regurgitation (PR). The sample volume is placed at the level of the pulmonary valve. RVOT, right ventricular outflow tract; $P A$, pulmonary artery; $A O$, aorta.

valves to image during echocardiography and there are few reports of vegetations of the pulmonary valve shown by this technique. We present an unusual case of endocarditis of the pulmonary valve that occurred in a patient with a normal heart. A vegetation was detected by cross sectional echocardiography. Because of recurrent pulmonary emboli the vegetation was excised.

\section{Case report}

A 25 year old man presented with a one week history of sweats and rigors. There was no history of drug abuse. There were no signs of endocarditis and examination of the cardiovascular system was normal. The resting electrocardiogram and chest radiographs were both normal. The haemoglobin concentration was $145 \mathrm{~g} / \mathrm{l}$, the white cell count was $7.5 \times 10^{9} / 1$, and the erythrocyte sedimentation rate was $45 \mathrm{~mm} /$ hour. Blood cultures grew Escherichia coli and Gram negative septicaemia was diagnosed. He was treated with gentamicin, penicillin, and metronidazole. On the fifth hospital day an early diastolic murmur of pulmonary regurgitation was heard and bacterial endocarditis was diagnosed.
Despite extensive investigations we found no cause for the underlying infection with $E$ coli. Echocardiography at this time was normal. He remained feverish and a second echocardiogram 18 days later showed a thickened pulmonary valve with a $2 \mathrm{~cm}$ echogenic mass associated with the valve. This was interpreted as a vegetation (fig 1 ). Pulmonary regurgitation was detected by Doppler ultrasound (fig 2). Subsequently episodes of pleuritic chest pain with haemoptysis developed and recurrent pulmonary infarction secondary to recurrent septic emboli was diagnosed. He therefore underwent exploration of the pulmonary valve and a large vegetation on the anterior leaflet was resected without valve replacement. His progress after operation was uneventful and his temperature returned to normal with antibiotic treatment. Six months later he was well and had a soft early diastolic murmur of pulmonary regurgitation.

\section{Discussion}

Endocarditis restricted to the right heart valves is uncommon. ${ }^{1}$ In the absence of congenital heart disease right sided infections are usually caused by a 
virulent organism or a large inoculation (such as occur during intravenous drug abuse) which usually affect the tricuspid valve rather than the pulmonary valve. The organism that most commonly causes right sided endocarditis is Staphylococcus aureus. ${ }^{1}$ Escherichia coli is rare but has been reported before. ${ }^{2}$ None the less, we are not aware of any previous reports of isolated pulmonary valve endocarditis caused by $E$ coli that occurred in the absence of the typical predisposing factors. Although virulent organisms are more commonly found in right sided endocarditis the course is less serious than left sided infection. ${ }^{1}$ Pneumonia or septic pulmonary emboli, however, are seen in $70-100 \%$ of cases of tricuspid endocarditis ${ }^{3}$ and Berger et al recently described three patients with pulmonary valve endocarditis who presented with pulmonary embolic phenomena." Operation for right sided endocarditis usually involves valve excision with or without implantation of a prosthetic valve. ${ }^{1}$ Resection of a vegetation without valve replacement has been performed in a case of tricuspid valve endocarditis, ${ }^{5}$ but not to our knowledge on a pulmonary valve. Because our patient was lean he was a suitable subject for echocardiography, but it is often difficult to show the pulmonary valve in adults. Like the patient described by Chambers and Leech, ${ }^{2}$ however, pulmonary regurgitation in our patient was easily detected by pulsed wave Doppler ultrasound. This may be a useful marker of pulmonary valve endocarditis in the absence of clear cross sectional echocardiographic images. The signal intensity was much greater than expected with physiological pulmonary regurgitation. We believe that a complete echocardiographic examination for endocarditis should include attempts to image the pulmonary valve and a Doppler ultrasound examination of the right ventricular outflow tract.

We thank Dr J Windebank, Dr P J B Hubner, and Mr $R \mathrm{~K}$ Firmin for permission to report a patient under their care.

\section{References}

1 Robbins MJ, Soeiro R, Frishman WH, Strom JA. Right-sided valvular endocarditis: etiology, diagnosis, and an approach to therapy. Am Heart J 1986;111:128-35.

2 Chambers J, Leech G. Echocardiographic demonstration of pulmonary valve endocarditis associated with congenital ventricular septal defect. $\mathrm{Br}$ Heart $\mathrm{J}$ 1986;55:494-6.

3 Reisberg BE. Infective endocarditis in the narcotic addict. Prog Cardiovasc Dis 1979;22:193-204.

4 Berger M, Wilkes MS, Gallerstein PE, Berdoff RL, Goldberg E. M-mode and two-dimensional echocardiographic findings in pulmonic valve endocarditis. Am Heart $J$ 1984;107:391-3.

5 Bernal JM, Gonzalez IM, Miralles PJ. Prophylactic resection of a tricuspid valve vegetation in infective endocarditis. Int J Cardiol 1986;12:255-7. 